\title{
20-Hydroxyecdysone ameliorates metabolic and cardiovascular dysfunction in high-fat- high-fructose-fed ovariectomized rats
}

\author{
Jariya Buniam ${ }^{1}$, Natsasi Chukijrungroat ${ }^{2}$, Yupaporn Rattanavichit ${ }^{3}$, Juthamard Surapongchai ${ }^{4}$, \\ Jittima Weerachayaphorn', Tepmanas Bupha-Intr ${ }^{1}$ and Vitoon Saengsirisuwan ${ }^{1 *}$ (D
}

\begin{abstract}
Background: Ecdysteroids are polyhydroxylated steroids present in invertebrates and plants. 20-Hydroxyecdysone (20E) is the most common and the main biologically active compound of ecdysteroids. Previous studies have demonstrated anabolic and metabolic effects of 20E in mammals. However, it is unknown whether 20E has a positive effect on all aspects of cardiometabolic syndrome. The aims of this study were to investigate the favorable effect and possible underlying mechanisms of $20 \mathrm{E}$ in a rat model of cardiometabolic syndrome (CMS) induced by a high-calorie diet combined with female sex hormone deprivation.
\end{abstract}

Methods: $20 \mathrm{E}(5 \mathrm{mg} / \mathrm{kg}, 10 \mathrm{mg} / \mathrm{kg}$, or $20 \mathrm{mg} / \mathrm{kg})$ or pioglitazone (PIO) $(10 \mathrm{mg} / \mathrm{kg})$ was intragastrically administered to sham-operated Sprague-Dawley female rats and ovariectomized rats fed a high-fat-high-fructose diet (OHFFD) for 8 weeks. The phenotypic characteristics of CMS, including central adiposity, blood pressure, serum lipid profile, glucose tolerance, insulin action on skeletal muscle glucose transport activity and hepatic protein expression, were determined.

Results: Some CMS characteristics were improved by 20E treatment. Rats treated with 20E had lower body weight, abdominal fat accumulation than rats treated with vehicle control without changes in total caloric intake and fatfree mass. OHFFD rats exhibited high blood pressure, but 20E-treated rats maintained normal blood pressure with a lower level of low-density lipoprotein (LDL)-cholesterol. Although 20E showed no positive effect on inducing insulin-mediated glucose transport in the skeletal muscle of OHFFD rats, 20E improved whole body glucose homeostasis. Analysis of protein expression in livers from 20E-treated rats revealed significantly increased expression of pAkt Ser ${ }^{473}$, pFOXO1 Ser ${ }^{256}$, pAMPKa Thr ${ }^{172}$, and FGF21.

Conclusion: 20E treatment can alleviate cardiometabolic disorder caused by a high-fat-high-fructose diet and female sex hormone deprivation. In particular, 20E helps improve whole body insulin sensitivity in OHFFD rats, and the mechanisms that underlie this favorable effect are potentially mediated by the activation of AMPK and FGF21. The present study indicates that $20 \mathrm{E}$ could be an alternative therapeutic option for the prevention and alleviation of cardiometabolic syndrome.

Keywords: 20-hydroxyecdysone, Ovariectomy, High-fat high-fructose diet, Glucose metabolism, Cardiometabolic syndrome

\footnotetext{
* Correspondence: vitoon.sae@mahidol.ac.th

'Department of Physiology, Faculty of Science, Mahidol University, Bangkok 10400, Thailand

Full list of author information is available at the end of the article
}

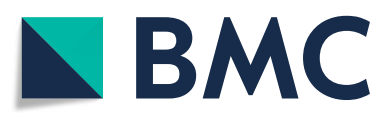

(c) The Author(s). 2020 Open Access This article is licensed under a Creative Commons Attribution 4.0 International License, which permits use, sharing, adaptation, distribution and reproduction in any medium or format, as long as you give appropriate credit to the original author(s) and the source, provide a link to the Creative Commons licence, and indicate if changes were made. The images or other third party material in this article are included in the article's Creative Commons licence, unless indicated otherwise in a credit line to the material. If material is not included in the article's Creative Commons licence and your intended use is not permitted by statutory regulation or exceeds the permitted use, you will need to obtain permission directly from the copyright holder. To view a copy of this licence, visit http://creativecommons.org/licenses/by/4.0/ The Creative Commons Public Domain Dedication waiver (http://creativecommons.org/publicdomain/zero/1.0/) applies to the data made available in this article, unless otherwise stated in a credit line to the data. 


\section{Background}

Cardiometabolic syndrome (CMS) is a clustering of multifaceted conditions characterized by several cardiovascular and metabolic risk factors, including dyslipidemia, hypertension, central obesity, compensatory hyperinsulinemia, and glucose intolerance $[1,2]$. One of the major causes of CMS is excessive caloric intake or alterations in diet composition. Fat intake appears to be an important determinant of obesity. In addition, an increase in fructose consumption, which is largely attributable to sweetened beverages, can lead to CMS [3-6]. Typically, the prevalence of CMS is higher in females after menopause $[7,8]$. However, currently, there are no specific medications that can prevent cardiometabolic dysfunction.

The pharmaceutical approach to CMS needs to be considered from various perspectives. Anti-diabetes agents are used as a broad base approach to induce whole-body insulin sensitivity and reduce the CMS phenotype. One of the most common clinical drugs prescribed to CMS patients is pioglitazone (PIO). Unfortunately, prolonged use of PIO may induce unfavorable effects, including edema, pulmonary edema, hepatic steatosis, and congestive heart failure [9, 10]. Thus, CMS medication discovery and development should preferably be shifted toward natural sources without side effects [9]. Notably, 20E is one of the candidates that has been studied [11-14]. Ecdysteroids are known as invertebrate steroid hormones that are involved in all stages of development, including newly laid eggs, embryogenic development, metamorphosis, reproduction and diapause [15]. Additionally, ecdysteroids can be found in many plant species around the world (phytoecdysteroids) [14, 15]. The major biologically active ecdysteroid found in invertebrates and plants is 20-hydroxyecdysone (20E) [12]. 20E is normally available in large amounts in certain plants, some of which are used for human food, such as spinach and quinoa [13]. A number of studies have revealed many potential beneficial health effects of $20 \mathrm{E}$ including the wound-healing, immunoprotective and anti-osteoporosis effects [12-15].

The early finding of the underlying mechanism was projected to be the anabolic effect of 20E [11]. For example, 20E was shown to increase protein synthesis in skeletal muscle [16] and increase physical endurance [17] in rodents. Furthermore, it has been reported that $20 \mathrm{E}$ is involved in metabolic effects in cell-based and animal-based studies $[14,15,18]$. The hypoglycemic [19, $20]$ and anti-obesity effects [21, 22] of 20E have been demonstrated in HepG2 cells and obese rodents, respectively. The findings on the metabolic effects of $20 \mathrm{E}$ revealed that $20 \mathrm{E}$ could prevent hyperglycemia in insulin-resistant rats by decreasing hepatic glucose consumption [19, 23]. More recent studies also showed that daily oral administration of $20 \mathrm{E}$ for 13 weeks prevents high-fat-diet-induced obesity [21, 24], whole body insulin resistance and hyperglycemia in mice by decreasing adipose depots, upregulating adiponectin expression in adipocytes, and modulating inflammatory adipokine expression [21]. However, relatively scarce literature is available on the properties of $20 \mathrm{E}$ as a treatment for multiple phenotypic characteristics of CMS. Importantly, there is still a lack of evidence showing whether $20 \mathrm{E}$ alleviates CMS features or glucose transport activity in insulin-resistant skeletal muscle. We have recently demonstrated that ovariectomized (OVX) rats fed a high-fathigh-fructose diet (OHFFD) represented a condition of menopausal women with CMS and skeletal muscle insulin resistance [25]. Therefore, this study was designed to investigate the effects of chronic treatment with $20 \mathrm{E}$ on insulin-mediated skeletal muscle glucose transport activity and other phenotypic characteristics of CMS in rats with metabolic dysfunction induced by a high-caloric diet after deprivation of female sex hormones.

\section{Methods \\ Animal studies}

Female Sprague-Dawley rats at 8 weeks of age, weighing approximately $180-200 \mathrm{~g}$, from the National Laboratory Animal Center, Mahidol University, Salaya, Thailand, were arrived on a strict hygienic conventional housing unit at the Center of Animal Facilities, Faculty of Science, Mahidol University. The temperature was maintained at $22^{\circ} \mathrm{C}$ with a $12 / 12$-h light/dark cycle. For concise determination of food and water intake, animals were individually housed in a $9 \times 12 \times 6$ in. hygienic hanging cage with corn cob bedding. Forty-six animals were randomly separated into 6 groups: sham $(n=8)$, OHFFD $(n=8)$, OHFFD treated with 20E $(5 \mathrm{mg} / \mathrm{kg}, 10$ $\mathrm{mg} / \mathrm{kg}, 20 \mathrm{mg} / \mathrm{kg}) \quad(n=7, n=8, n=8)$, and OHFFD treated with PIO $(n=7)$. The sample size was calculated from insulin-mediated glucose transport data according to Prasannarong et al., 2012 [26] by using Minitab 14 (Minitab Inc., State College, PA, USA). The baseline data on blood pressure, body weight, and food and water intake of each animal were recorded. During the experimental period, the amount of food and water consumed as well as the body weight of each animal were measured. At 10 weeks of age, ovariectomy or sham surgical procedures were performed under anesthesia through bilateral skin incisions at the lower back, as previously described [27]. A mixture of Tiletamine-zolazepam (Zoletil $50 ; 25 \mathrm{mg} / \mathrm{kg}$ body weight) plus xylazine (3-5 $\mathrm{mg} / \mathrm{kg}$ body weight) was intraperitoneally injected to put the animals into deep anesthesia. Provision of analgesic drug was used for one week after the surgery. Sham rats were fed a control diet (TD08806, 10\% kcal as fat; Envigo 
[formerly Harlan Teklad], Cambridgeshire, UK) with reverse osmosis water. OVX rats were fed a high-fat diet (TD06414, 60\% kcal as fat; Envigo) with 30\% w/v fructose in drinking water for 12 weeks ad libitum. From the 4th week of the experiment, the treated group was daily given $20 \mathrm{E}(5 \mathrm{mg} / \mathrm{kg}, 10 \mathrm{mg} / \mathrm{kg}, 20 \mathrm{mg} / \mathrm{kg})$ or PIO $(10 \mathrm{mg} / \mathrm{kg})$ diluted in $25 \%$ propylene glycol by gavage at $0900-1000 \mathrm{~h}$. These concentrations of $20 \mathrm{E}$ had been used in previous studies [19, 21, 23]. The animals that received vehicle treatment were orally administered $25 \%$ propylene glycol (TCI, Tokyo, Japan). Body weight, food intake and water intake were assessed 3 times a week throughout the 12 weeks of feeding. The total caloric intake of each animal was calculated using the average daily food and water (or fructose solution) consumed and multiplying by the calorie content of the respective diet.

\section{Blood pressure measurement}

A noninvasive computerized tail-cuff system (CODA Monitor System, Kent Scientific Corporation, Torrington, $\mathrm{CT}$ ) with the indirect tail-cuff method was used to measure systolic blood pressure (SBP), diastolic blood pressure (DBP) and mean arterial pressure (MAP) at baseline and at the 12th week of the experiment in conscious rats. The reported values for each rat were the mean of 10 consecutive readings.

\section{Determination of whole-body insulin sensitivity}

After an 11-week period of treatment, an oral glucose tolerance test (OGTT) was performed. Approximately $15 \mathrm{~h}$ before the test, all animals were food restricted ( $4 \mathrm{~g}$ of food), and the fructose solution was replaced with reverse-osmosis water. On the day of the test, $24 \mathrm{~h}$ after the last treatment, tail blood was collected before glucose feeding ( $1 \mathrm{~g} / \mathrm{kg}$ body weight) by gavage and $15,30,60$, and $120 \mathrm{~min}$ after the glucose challenge. Blood samples were mixed with the anticoagulant EDTA and centrifuged at $13,000 \mathrm{~g}$ and $4{ }^{\circ} \mathrm{C}$ for 1 min. Plasma was kept at $80^{\circ} \mathrm{C}$ and used for the determination of glucose (Gesellschaft für Biochemica und Diagnostica, Wiesbaden, Germany) and insulin (Linco Research, MO, USA). Immediately after the OGTT, each animal was given $2.5 \mathrm{ml}$ of sterile $0.9 \%$ saline to substitute the lost fluid. The homeostatic model assessment of insulin resistance (HOMA-IR) score was calculated to reflect the whole-body insulin sensitivity from fasting glucose and fasting insulin at baseline. The glucose-insulin (G-I) index was calculated as the product of glucose and insulin areas under the curves (AUCs) and was used to indicate whole-body insulin sensitivity, with a high G-I index indicating low whole-body insulin sensitivity.

\section{Blood and tissue collection}

All animals were food restricted ( $4 \mathrm{~g}$ of food), and the fructose solution was replaced with reverse osmosis water $15 \mathrm{~h}$ before euthanasia. Rats were weighed and then anesthetized with an intraperitoneal injection of pentobarbital sodium (nembutal; $75 \mathrm{mg} / \mathrm{kg}$ body weight). Soleus muscles were isolated and prepared for in vitro assessment of insulin-mediated muscle glucose transport activity. The plantaris muscle was collected and weighed for representation of lean body mass. Blood was drawn from the abdominal artery, allowed to clot, and centrifuged at $3000 \mathrm{~g}$ and $4{ }^{\circ} \mathrm{C}$ for $15 \mathrm{~min}$ to obtain serum samples. The liver and visceral fat pads were excised and immediately weighed. All liver samples were collected from the same lobe of the liver for each animal and were frozen in liquid nitrogen. The uterus was removed and weighed for confirmation of a complete ovariectomy, and the heart was removed to euthanize the animals. Serum and tissue samples were stored at $-80^{\circ} \mathrm{C}$ until analysis.

\section{Assessment of insulin-mediated muscle glucose transport activity}

Insulin action on glucose transport activity was determined in insulin-mediated conditions using 2-deoxy$[3 \mathrm{H}]$-glucose (2-DG) uptake. The soleus muscle was isolated, divided into 3 portions, and incubated for $60 \mathrm{~min}$ at $37^{\circ} \mathrm{C}$ in $3 \mathrm{ml}$ of oxygenated Krebs-Henseleit buffer (KHB) supplemented with $8 \mathrm{mM}$ D-glucose, $32 \mathrm{mM}$ Dmannitol and $0.1 \%$ radioimmunoassay-grade bovine serum albumin (BSA) (Sigma Chemical, MO, USA). One strip of the soleus muscle was incubated in the absence of insulin, and the other strip was incubated in the presence of $0.2 \mathrm{mU} / \mathrm{ml}$ and $2 \mathrm{mU} / \mathrm{ml}$ insulin (Human R; Eli Lilly, IN, USA). Flasks were continuously gassed with a mixture of $95 \% \mathrm{O}_{2}$ and $5 \% \mathrm{CO}_{2}$ throughout the incubation and transport study procedures. After the first incubation period, each muscle strip was rinsed for $10 \mathrm{~min}$ at $37^{\circ} \mathrm{C}$ in $3 \mathrm{ml}$ of oxygenated $\mathrm{KHB}$ containing $40 \mathrm{mM} \mathrm{D}$ mannitol, $0.1 \%$ BSA and insulin (for the muscle strip incubated in the presence of insulin). Each muscle strip was then incubated for $20 \mathrm{~min}$ at $37^{\circ} \mathrm{C}$ in $2 \mathrm{ml}$ of $\mathrm{KHB}$ containing $1 \mathrm{mM} \quad 2-\left[1,2-{ }^{3} \mathrm{H}\right]$ deoxyglucose (2-DG) $300 \mu \mathrm{Ci} / \mathrm{mmol}$; PerkinElmer Life Sciences, Boston, MA), $39 \mathrm{mM} \quad\left[\mathrm{U}_{-}{ }^{14} \mathrm{C}\right]$ mannitol $\quad(0.8 \mu \mathrm{Ci} / \mathrm{mmol}$, PerkinElmer Life Sciences), $0.1 \%$ BSA and insulin (for the muscle strip incubated in the presence of insulin). At the end of the incubation period, muscle strips were removed and trimmed of excess fat and connective tissue, immediately frozen with liquid nitrogen and weighed. Frozen muscles were solubilized in $0.5 \mathrm{ml}$ of $0.5 \mathrm{M} \mathrm{NaOH}$, and $8 \mathrm{ml}$ of scintillation cocktail was added (Ultima Gold; PerkinElmer Life Sciences). Specific intracellular accumulation of 2-DG was determined as previously described [28] using 
mannitol to correct for extracellular accumulation of 2DG. Glucose transport activity was measured by determination of the intracellular accumulation of 2-DG ( $\mathrm{pmol} / \mathrm{mg}$ muscle wet weight $/ 20 \mathrm{~min}$ ). Insulin-mediated glucose transport was calculated as the net increase in 2DG uptake above the basal level due to insulin.

\section{Immunoblotting analysis}

Frozen rat liver tissue $(50 \mathrm{mg})$ was homogenized using a TissueLyser LT (QIAGEN, Valencia, CA, USA) in $500 \mu \mathrm{l}$ of ice-cold RIPA lysis buffer (Thermo Scientific Inc., Waltham, MA, USA) containing Halt protease and phosphatase inhibitor cocktail (Thermo Scientific Inc.). Liver homogenates were centrifuged at $12,000 \mathrm{~g}$ and $4{ }^{\circ} \mathrm{C}$ for 20 $\mathrm{min}$, and the protein concentration was determined using the BCA Protein Assay Kit (Pierce, Rockford, IL, USA). Fifty micrograms of protein from each sample was separated using 8-10\% SDS-PAGE and blotted onto $0.45 \mu \mathrm{m}$ nitrocellulose membranes (Bio-Rad, Richmond, CA, USA). Blots were blocked with $5 \%$ Omniblok nonfat dry milk (AmericanBio, Inc., MA, USA) in tris-buffered saline (TBS) plus $0.1 \%$ Tween- 20 for $2 \mathrm{~h}$ and incubated with the following primary antibodies at $4{ }^{\circ} \mathrm{C}$ overnight: FOXO1 (1: 1000, Santa Cruz Biotechnology, TX, USA), pFOXO1 $\operatorname{Ser}^{256}$ (1:1000, Santa Cruz Biotechnology), Akt (1:800), pAkt $\operatorname{Ser}^{473}$ (1:800), AMPK $\alpha$ (1:800), pAMPK $\alpha \operatorname{Thr}^{172}$ (1: 800), FGF21 (1:1000, Abcam, Cambridge, UK) and GAPDH (1:3000). Blots were subsequently incubated with horseradish peroxidase (HRP)-conjugated goat anti-rabbit IgG (1:3000) or HRP-conjugated goat-anti mouse IgG secondary antibody (1:3000) at room temperature for $60 \mathrm{~min}$. Antibodies without a supplier indicated were purchased from Cell Signaling Technology (Beverly, MA, USA). Protein bands were visualized by enhanced chemiluminescence (PerkinElmer Life Sciences) using a C-DiGit Blot Scanner (LI-COR Biotechnology, Lincoln, NE, USA) and were quantified with Image Studio software. Band densities were quantified using ImageJ software (NIH). GAPDH was used as an internal control for normalizing protein expression.

\section{Statistical analysis}

The significance of differences among groups was assessed by one-way analysis of variance (ANOVA) with a post hoc Tukey honest significant difference test. A level of $P<0.05$ was set to indicate statistical significance.

\section{Results}

\section{Effect of 20E on total caloric intake and body weight}

The OHFFD rats consumed more calories than the sham rats $(+40 \%, P<0.05)$, but there was no significant difference among the 20E-treated groups (Table 1). The final body weight and total body weight gain were also higher in the OHFFD group $(+33 \%,+158 \%, P<0.05$, Table 1$)$ than in the sham group. 20E reduced body weight in a dose-dependent manner but was only significant in the $20 \mathrm{E} 20 \mathrm{mg} / \mathrm{kg}$ group $(-16 \%, P<0.05)$. This indicated that $20 \mathrm{E}$ has a beneficial effect on controlling body weight without affecting eating habits.

\section{Effects of 20E on tissue weights}

Estrogen plays a critical role in the regulation of the maturational process of female reproductive organs [29]. Uterus weights in the OHFFD rats were 5-fold lower than that of the sham rats, indicating the effectiveness of the ovariectomy. The uterus weights of the OHFFD rats were lower than those of the sham rats and showed no improvement in 20E-treated rats (Table 1), suggesting that 20E treatment had no estrogenic effect on uterus weight. Central obesity was estimated by the ratio of abdominal fat weight to body weight. When compared to the sham rats, the OHFFD rats had $a+21 \%$ increase in abdominal fat mass $(P<0.05)$, which was suppressed by $20 \mathrm{E}$ treatment in both the $10 \mathrm{mg} / \mathrm{kg}$ and $20 \mathrm{mg} / \mathrm{kg}$ groups ($18 \%,-16 \%, P<0.05$, Table 1$)$. However, no significant difference in the ratio of plantaris muscle weight to body weight was observed among the groups.

\section{Effect of $20 \mathrm{E}$ on serum lipid profile}

Serum lipid profiles are shown in Table 2. The levels of serum triglycerides were significantly increased by $+96 \%$ in the OHFFD rats $(P<0.05)$, and PIO reduced the circulating levels of triglycerides. No significant differences were detected in the levels of total serum cholesterol (TC) among the groups. The OHFFD rats exhibited significant increases in LDL cholesterol $(+46 \%, P<0.05)$ and the LDL/TC ratio $(+58 \%, P<0.05)$ when compared to the sham animals. $20 \mathrm{E}(10 \mathrm{mg} / \mathrm{kg}$ and $20 \mathrm{mg} / \mathrm{kg})$ and PIO treatments reduced the levels of serum LDL cholesterol $(-21 \%,-26 \%,-15 \%, P<0.05)$ and the LDL/TC ratio $(-15 \%,-15 \%,-10 \%, P<0.05)$ in the OHFFD rats. There were no significant differences in the levels of HDL cholesterol or the HDL/TC ratio among the groups.

\section{Effect of 20E on arterial blood pressure}

Table 3 demonstrates systolic blood pressure (SBP), diastolic blood pressure (DBP) and mean arterial pressure (MAP) at baseline and at 12 weeks, i.e., at the end of the interventions. The OHFFD rats exhibited a significant increase in SBP, DBP and MAP $(+26 \%, P<0.05)$ compared with the same parameters in the sham rats. $20 \mathrm{E}$ and PIO evidently normalized blood pressure in the OHFFD rats $(P<0.05$, Table 3$)$. 
Table 1 Initial and final body weights; BW gain; total caloric intake; uterus, liver, and abdominal fat weights; plantaris muscle weight; fasting plasma insulin; fasting plasma glucose; and HOMA-IR score in sham rats fed a control diet and OVX rats fed a HFFD (OHFFD) that received 20-hydroxyecdysone (20E) or pioglitazone (PIO)

\begin{tabular}{|c|c|c|c|c|c|c|}
\hline & SHAM & OHFFD & $\begin{array}{l}\text { OHFFD + 20E } \\
(5 \mathrm{mg} / \mathrm{kg} \mathrm{Bw}) \\
\end{array}$ & $\begin{array}{l}\text { OHFFD + 20E } \\
(10 \mathrm{mg} / \mathrm{kg} \mathrm{Bw})\end{array}$ & $\begin{array}{l}\text { OHFFD + 20E } \\
(20 \mathrm{mg} / \mathrm{kg} \mathrm{Bw})\end{array}$ & $\begin{array}{l}\text { OHFFD + PIO } \\
(10 \mathrm{mg} / \mathrm{kg} \mathrm{Bw})\end{array}$ \\
\hline \multicolumn{7}{|l|}{ Body weight (BW), g } \\
\hline Initial BW & $225.8 \pm 2.3$ & $221.6 \pm 3.0$ & $212.0 \pm 1.3$ & $215.5 \pm 3.4$ & $215.3 \pm 4.7$ & $208.9 \pm 1.2$ \\
\hline Final BW & $292.9 \pm 3.7$ & $390.4 \pm 5.6^{\#}$ & $366.3 \pm 11.5^{\#}$ & $366.3 \pm 5.1^{\#}$ & $361.4 \pm 8.5^{\# *}$ & $370.9 \pm 8.4^{\#}$ \\
\hline BW gain, $g$ & $61.9 \pm 3.1$ & $158.9 \pm 5.2^{\#}$ & $156.9 \pm 9.6^{\#}$ & $152.6 \pm 5.6^{\#}$ & $133.4 \pm 4.8^{\# *}$ & $161.4 \pm 6.9^{\#}$ \\
\hline Total caloric intake, kcal & $4394 \pm 67$ & $6163 \pm 118^{\#}$ & $6044 \pm 164^{\#}$ & $6377 \pm 164^{\#}$ & $6288 \pm 199^{\#}$ & $6160 \pm 190^{\#}$ \\
\hline Uterus weight, mg & $741 \pm 40.2$ & $92 \pm 2.4^{\#}$ & $91 \pm 2.4^{\#}$ & $96 \pm 4.4^{\#}$ & $93 \pm 3.5^{\#}$ & $85 \pm 5.2^{\#}$ \\
\hline Liver weight, g & $6.7 \pm 0.1$ & $8.5 \pm 0.2^{\#}$ & $8.8 \pm 0.2^{\#}$ & $8.8 \pm 0.3^{\#}$ & $9.0 \pm 0.3^{\#}$ & $7.8 \pm 0.4^{\#}$ \\
\hline Abdominal fat weight/ body weight, $\%$ & $5.7 \pm 0.2$ & $6.9 \pm 0.3^{\#}$ & $6.3 \pm 0.4^{\#}$ & $5.7 \pm 0.3^{*}$ & $5.8 \pm 0.1^{*}$ & $5.6 \pm 0.3^{*}$ \\
\hline Plantaris weight/ body weight, $\%$ & $0.92 \pm 0.12$ & $0.89 \pm 0.15$ & $0.86 \pm 0.03$ & $0.87 \pm 0.02$ & $0.86 \pm 0.02$ & $0.82 \pm 0.02$ \\
\hline Fasting plasma glucose, mg/dl & $123.3 \pm 2.3$ & $128 \pm 4.4$ & $127.2 \pm 3.5$ & $125.3 \pm 2.4$ & $131.8 \pm 1.8$ & $118.5 \pm 1.7$ \\
\hline Fasting plasma insulin, $\mu \mathrm{U} / \mathrm{ml}$ & $52.7 \pm 4.8$ & $61 \pm 4.4$ & $61.4 \pm 8.2$ & $58.7 \pm 7.6$ & $59.4 \pm 5.5$ & $30.5 \pm 4.3^{*}$ \\
\hline HOMA IR score & $1.6 \pm 0.1$ & $1.9 \pm 0.1^{\ddagger}$ & $1.9 \pm 0.3^{\ddagger}$ & $1.8 \pm 0.2^{\ddagger}$ & $1.9 \pm 0.2^{\ddagger}$ & $0.9 \pm 0.1$ \\
\hline
\end{tabular}

Values are means \pm SEMs for 7-8 animals/group

Data for comparisons between groups were analyzed using one-way ANOVA followed by Tukey's post hoc analysis. ${ }^{\sharp} P<0.05$ vs. sham; ${ }^{*} P<0.05$ vs. OHFFD, ${ }^{\ddagger} P<$ 0.05 vs. OHFFD+PIO $(10 \mathrm{mg} / \mathrm{kg})$

\section{Effect of 20E on whole body insulin sensitivity}

The levels of plasma glucose and insulin during fasting are shown in Table 1 . There was no significant difference in fasting plasma glucose among the groups. However, fasting plasma insulin showed a significant decrease in the OHFFD+PIO group $(10 \mathrm{mg} / \mathrm{kg})$ compared to that in the OHFFD group $(-51 \%, P<0.05)$. The 20E-treated groups showed no significant difference in fasting insulin levels. The HOMA-IR score of each rat was calculated based on the relationship between fasting plasma glucose and insulin. The results showed an improvement in insulin sensitivity in PIO-treated rats compared to other OHFFD groups $(P<0.05)$. The levels of plasma glucose and insulin during the OGTTs are shown in Fig. 1. After the glucose challenge, the OHFFD+20E $(20 \mathrm{mg} / \mathrm{kg})$ rats exhibited significant decreases in plasma glucose levels at the 30-min and 60- min time points compared to the levels in the OHFFDgroup (Fig. 1a), with a $10 \%$ decrease in the area under the curve for glucose (glucose AUC) $(P<0.05$; Fig. 1c) compared with the AUC of the OHFFD rats. Moreover, the insulin levels at the 15-, 30-, and 60-min time points in the OHFFD rats were significantly higher than those of the sham rats (Fig. 1b). Additionally, there was a $66 \%$ increase in the insulin AUC in OHFFD group $(P<0.05$; Fig. 1d) compared to that of the sham rats. 20E treatment at a dose of $20 \mathrm{mg} / \mathrm{kg}$ and PIO significantly decreased the insulin AUC by $33 \%$ and $60 \%$, respectively, compared with that of the OHFFD rats. The glucoseinsulin (G-I) index, the product of the glucose and insulin AUCs, is inversely related to an increase in whole body insulin sensitivity. The G-I index in the OHFFD rats was higher than that in any other group, indicating impaired glucose tolerance and whole-body insulin

Table 2 Triglycerides, total cholesterol (TC), LDL-cholesterol (LDL), HDL-cholesterol (HDL), LDL/TC, and HDL/TC in sham rats fed a control diet and OVX rats fed a HFFD (OHFFD) that received 20-hydroxyecdysone (20E) or pioglitazone (PIO)

\begin{tabular}{lllllll}
\hline & SHAM & OHFFD & $\begin{array}{l}\text { OHFFD }+20 E \\
(5 \mathrm{mg} / \mathrm{kg} \mathrm{Bw})\end{array}$ & $\begin{array}{l}\text { OHFFD + 20E } \\
(10 \mathrm{mg} / \mathrm{kg} \mathrm{Bw})\end{array}$ & $\begin{array}{l}\text { OHFFD + 20E } \\
(20 \mathrm{mg} / \mathrm{kg} \mathrm{Bw})\end{array}$ & $\begin{array}{l}\text { OHFFD + PIO } \\
(10 \mathrm{mg} / \mathrm{kg} \mathrm{Bw})\end{array}$ \\
\hline Triglycerides, $\mathrm{mg} / \mathrm{mL}$ & $29.0 \pm 1.7$ & $57.0 \pm 6.5^{\#}$ & $41.9 \pm 27.7$ & $34.1 \pm 10.5$ & $36.4 \pm 4.3$ & $26.5 \pm 3.3^{*}$ \\
Total cholesterol, $\mathrm{mg} / \mathrm{dL}$ & $102.5 \pm 2.5$ & $103.0 \pm 2.2$ & $90.7 \pm 1.7$ & $92.5 \pm 2.3$ & $97.0 \pm 5.1$ & $130.78 \pm 3.4$ \\
LDL-cholesterol, $\mathrm{mg} / \mathrm{dL}$ & $12.7 \pm 0.3$ & $19.3 \pm 0.5^{\#}$ & $18.4 \pm 0.8^{\#}$ & $15.1 \pm 0.4^{*}$ & $14.9 \pm 0.5^{*}$ & $16.0 \pm 0.7^{\# *}$ \\
HDL-cholesterol, $\mathrm{mg} / \mathrm{dL}$ & $83.0 \pm 2.0$ & $85.3 \pm 2.2$ & $82.1 \pm 2.0$ & $77.9 \pm 2.2$ & $77.7 \pm 5.1$ & $78.0 \pm 1.9$ \\
LDL/TC & $0.12 \pm 0.002$ & $0.19 \pm 0.004^{\#}$ & $0.20 \pm 0.010^{\#}$ & $0.16 \pm 0.004^{\dagger}$ & $0.16 \pm 0.010^{*}$ & $0.17 \pm 0.006^{*}{ }^{\dagger}$ \\
HDL/TC & $0.81 \pm 0.03$ & $0.83 \pm 0.02$ & $0.91 \pm 0.02$ & $0.84 \pm 0.3$ & $0.80 \pm 0.03$ & $0.81 \pm 0.03$ \\
\hline
\end{tabular}

Values are means \pm SEMs for 7-8 animals/group

Data for comparisons between groups were analyzed using one-way ANOVA followed by Tukey's post hoc analysis. ${ }^{\sharp} P<0.05$ vs. sham; ${ }^{*} P<0.05$ vs. OHFFD; ${ }^{\dagger} P<$ 0.05 vs. OHFFD+20E $(5 \mathrm{mg} / \mathrm{kg})$ 
Table 3 Systolic blood pressure, diastolic blood pressure and mean arterial pressure in sham rats fed a control diet and OVX rats fed a HFFD (OHFFD) that received 20-hydroxyecdysone (20E) or pioglitazone (PIO)

\begin{tabular}{|c|c|c|c|c|c|c|}
\hline & SHAM & OHFFD & $\begin{array}{l}\text { OHFFD + 20E } \\
(5 \mathrm{mg} / \mathrm{kg} \text { BW })\end{array}$ & $\begin{array}{l}\text { OHFFD + 20E } \\
(10 \mathrm{mg} / \mathrm{kg} \mathrm{BW})\end{array}$ & $\begin{array}{l}\text { OHFFD + 20E } \\
(20 \mathrm{mg} / \mathrm{kg} \mathrm{BW})\end{array}$ & $\begin{array}{l}\text { OHFFD + PIO } \\
(10 \mathrm{mg} / \mathrm{kg} \text { BW })\end{array}$ \\
\hline \multicolumn{7}{|l|}{$\mathrm{SBP}, \mathrm{mmHg}$} \\
\hline Baseline & $119.7 \pm 2.4$ & $125.9 \pm 1.8$ & $123.7 \pm 1.0$ & $122.9 \pm 1.6$ & $126.9 \pm 1.3$ & $119.9 \pm 1.4$ \\
\hline Week 12 & $119.7 \pm 1.3$ & $153.4 \pm 3.1^{\#}$ & $136.6 \pm 2.8^{*}$ & $128.7 \pm 3.0^{*}$ & $129.1 \pm 2.7^{*}$ & $130.8 \pm 5.1^{*}$ \\
\hline \multicolumn{7}{|l|}{$\mathrm{DBP}, \mathrm{mmHg}$} \\
\hline Baseline & $87.8 \pm 4.1$ & $92.2 \pm 4.1$ & $92.7 \pm 1.3$ & $94.5 \pm 2.3$ & $96.8 \pm 1.7$ & $86.2 \pm 1.7$ \\
\hline Week 12 & $86.1 \pm 3.8$ & $114.6 \pm 2.8^{\#}$ & $108.5 \pm 2.8^{*}$ & $99.7 \pm 2.5^{*}$ & $98.7 \pm 3.8^{*}$ & $98.2 \pm 5.9^{*}$ \\
\hline \multicolumn{7}{|c|}{ MAP, mmHg } \\
\hline Baseline & $98.5 \pm 3.6$ & $100.6 \pm 2.6$ & $104.9 \pm 1.7$ & $104.3 \pm 1.7$ & $106.5 \pm 1.5$ & $98.4 \pm 1.1$ \\
\hline Week 12 & $96.9 \pm 2.8$ & $126.9 \pm 2.6^{\#}$ & $115.3 \pm 3.8^{*}$ & $109.1 \pm 2.6^{*}$ & $107.1 \pm 3^{*}$ & $108.6 \pm 3.1^{*}$ \\
\hline
\end{tabular}

Values are means \pm SEMs for 7-8 animals/group

Data for comparisons between groups were analyzed using one-way ANOVA followed by Tukey's post hoc analysis. ${ }^{\#} P<0.05$ vs. sham; ${ }^{*} P<0.05$ vs. OHFFD

resistance. The G-I index in the OHFFD rats was effectively attenuated by $20 \mathrm{E}$ supplementation and PIO treatment $(P<0.05$; Fig. $1 \mathrm{e})$.

\section{Effect of $20 \mathrm{E}$ on signaling pathway proteins involved in gluconeogenesis in the liver}

As FOXO1, Akt, AMPK $\alpha$, and FGF21 are signaling components contributing to gluconeogenesis in hepatic cells, the activity of these proteins in the liver was evaluated (Fig. 2). The analysis showed no significant difference in the protein expression of Akt, FOXO1, or AMPKa in any groups, as well as no significant difference in the protein expression of pAkt $\mathrm{Ser}^{473}$ or pFOXO1 $\mathrm{Ser}^{256}$ in OHFFD rats compared to that in sham rats. However, there were significant differences in the protein expression of pAMPK $\alpha$ $\mathrm{Thr}^{172} / \mathrm{AMPK} \alpha(-56 \%, P<0.05)$ and FGF21 $(-76 \%$, $P<0.05)$ between the OHFFD and sham groups. Although there was no beneficial effect observed in the $20 \mathrm{E}(5 \mathrm{mg} / \mathrm{kg})$ treatment group compared to the OHFFD group, the $20 \mathrm{E}$ at $10 \mathrm{mg} / \mathrm{kg}$ group exhibited increased phosphorylation of FOXO1 $\mathrm{Ser}^{256} / \mathrm{FOXO} 1$ $(+182 \%, \quad P<0.05)$ and AMPK $\alpha \mathrm{Thr}^{172} / \mathrm{AMPK} \alpha \quad(+$ $147 \%, P<0.001)$ and protein expression of FGF21 (+ $184 \%, P<0.05)$. The $20 \mathrm{E}$ at $20 \mathrm{mg} / \mathrm{kg}$ treatment was proven to further increase the activity of pFOXO1 Ser ${ }^{256} /$ FOXO1 $(+267 \%, \quad P<0.01), \quad$ pAMPK $\alpha \mathrm{Thr}^{172} /$ AMPKo $(+262 \%, P<0.001)$, and FGF21 $(+468 \%), P<$ 0.01 ) and showed a significant difference in pAkt Ser ${ }^{473} /$ Akt $(+144 \%, P<0.05)$ compared to that in the OHFFD group.

Effect of $20 \mathrm{E}$ on insulin-mediated skeletal muscle glucose transport activity

Insulin-mediated glucose transport represents insulin action on glucose transport activity in skeletal muscle, which was calculated as the net increase in glucose uptake above the basal level due to insulin stimulation. There was a significant reduction in insulinmediated skeletal muscle glucose transport at the 0.2 $\mathrm{mU} / \mathrm{ml}$ insulin concentrations in the PIO-treated group compared to that in the sham group $(P<0.05$, Fig. 3a). In addition, there was a decrease in insulin-mediated skeletal muscle glucose transport in the $2.0 \mathrm{mU} / \mathrm{ml}$ insulin concentration of OHFFD group $(-40 \%, P<0.05$, Fig. 3b). Nonetheless, no beneficial effects were found in insulin-mediated skeletal muscle glucose transport activity in 20E- or PIOtreated rats. It should be noted that the glucose transport data was excluded if the animals died prior to muscle dissection.

\section{Discussion}

The present study demonstrated that 20E has a favorable effect on whole-body glucose tolerance and alleviates CMS phenotypic characteristics in an HFFD-induced metabolic disorder rat model. This investigation provided novel information that $20 \mathrm{E}$ can improve whole body insulin sensitivity in OHFFD rats, and the mechanism may involve the liver. 20E increased hepatic function in glucose homeostasis by activating the protein expression of pAkt $\mathrm{Ser}^{473}$, pFOXO1 Ser ${ }^{256}$, pAMPK $\alpha \mathrm{Thr}^{172}$, and FGF21. Our study was the first to identify the metabolic effect of $20 \mathrm{E}$ on skeletal muscle glucose transport in CMS rats induced by a high-caloric diet after deprivation of female sex hormones. Importantly, this study demonstrated that 20E did not modulate insulin-mediated glucose transport activity in skeletal muscle of OHFFD rats, indicating that the favorable effect of $20 \mathrm{E}$ on whole-body insulin sensitivity is not associated with skeletal muscle function. Additionally, we demonstrated that a high dose of $20 \mathrm{E}$ treatment reduced CMS characteristics, including abdominal fat mass, LDL cholesterol levels, and blood pressure.

In the present study, the OHFFD rat model represented a condition of menopausal women with CMS, 

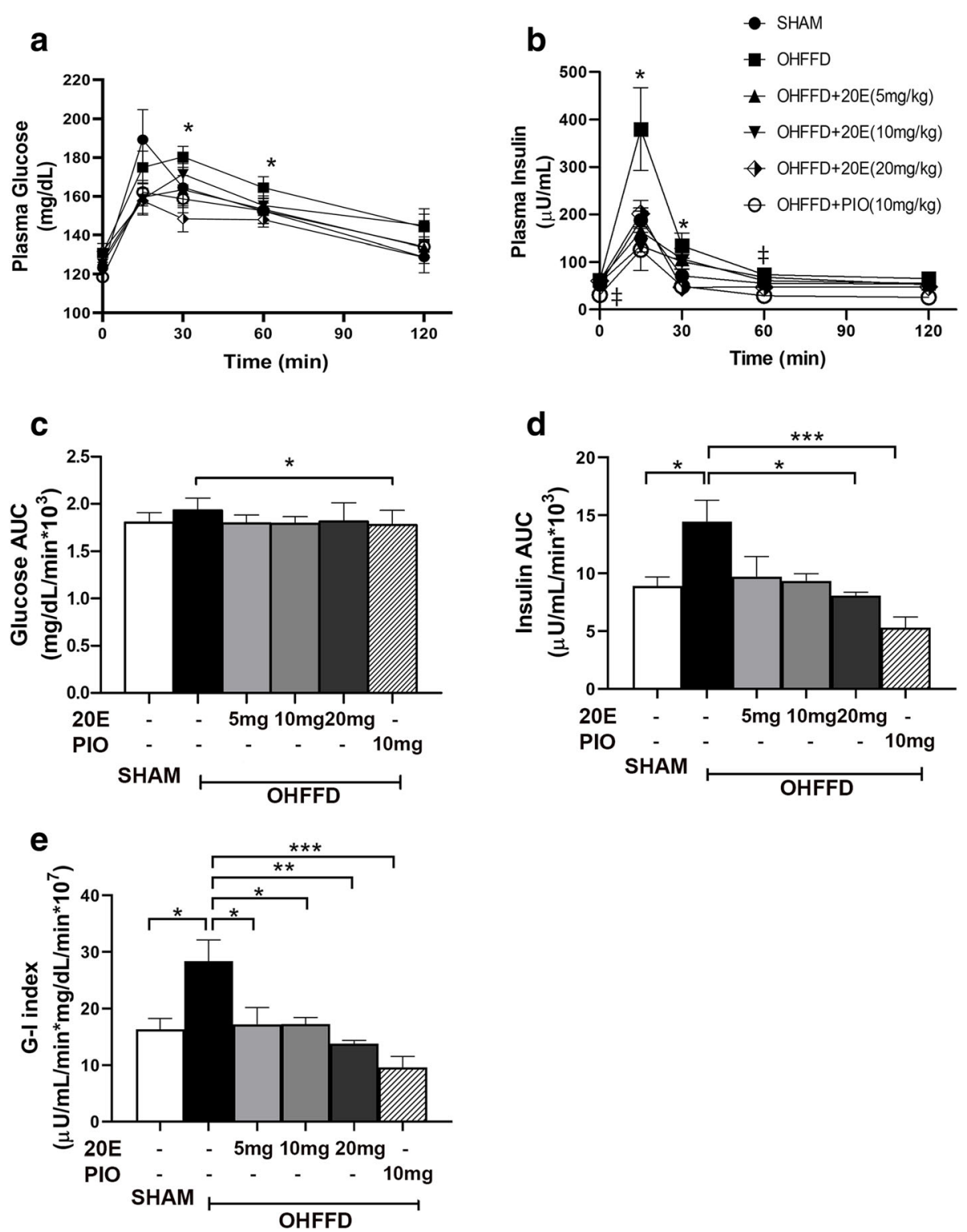

Fig. 1 Plasma glucose (a) and insulin (b) during an oral glucose tolerance test (OGTT), area under the curve (AUC) for glucose (c) and insulin (d), and glucose-insulin (G-I) index in sham-operated (sham) rats fed a control diet and ovariectomized rats fed a high-fat high-fructose diet (OHFFD) that received 20-hydroxyecdysone (20E) and pioglitazone (PIO). Data for the AUCs were calculated from the glucose and insulin responses. The GI index was the product of the glucose AUC and insulin AUC for each individual animal. Values are means \pm SE for 7-8 animals/group. Data comparisons between groups were performed using one-way ANOVA followed by Tukey's post hoc analysis. ${ }^{*} P<0.05$ vs. OHFFD, $\neq P<0.05$ PIO vs. OHFFD

including dyslipidemia, hypertension, central obesity, and impaired glucose tolerance along with other characteristics of metabolic abnormalities, such as insulin resistance of skeletal muscle glucose transport activity. The major finding indicated that 20E can improve whole body insulin sensitivity and reduce hyperinsulinemia (Fig. 1). The improvement in whole-body glucose tolerance may be accompanied in part by an increase in the function of insulin-sensitive organs. Several studies in
HepG2 [19] and H4IIE [23] cells revealed that 20E can increase hepatic function by increasing hepatic glucose uptake and reduce hepatic glucose production by downregulating PEPCK and G6Pase gene expression through the activation of the insulin-stimulated PI3K/Akt pathway $[19,23,30]$. These two proteins are key enzymes in the hepatic gluconeogenesis pathway. Our findings confirmed that 20E increased hepatic function by decreasing hepatic gluconeogenesis through both an insulin- 


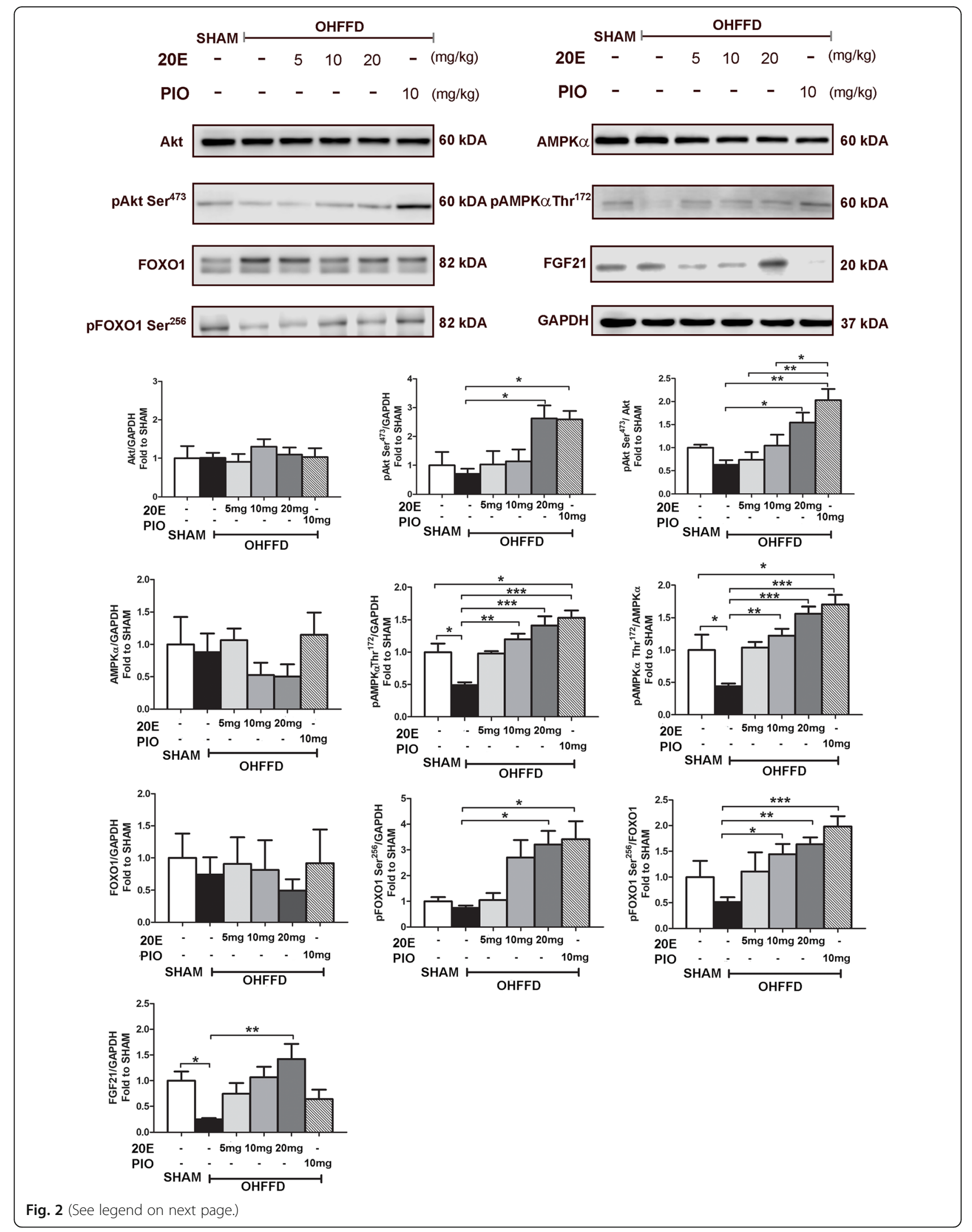


(See figure on previous page.)

Fig. 2 Effects of 20-hydroxyecdysone (20E; $5 \mathrm{mg} / \mathrm{kg}, 10 \mathrm{mg} / \mathrm{kg}, 20 \mathrm{mg} / \mathrm{kg}$ ) and pioglitazone (PIO) on the levels of the following signaling proteins in the liver: Akt, pAkt Ser ${ }^{473}$, FOXO1, pFOXO1 Ser ${ }^{256}$, AMPKa, pAMPKa Thr ${ }^{172}$, and FGF21. The signaling proteins were measured by immunoblotting and normalized to GAPDH. Data are listed as the fold-change in expression levels relative to the sham control group. Representative bands are shown in the top panel. OHFFD: OVX fed a high-fat high-fructose diet. Values are means \pm SEM for 7-8 animals/group. Data comparisons between groups were performed using one-way ANOVA followed by Tukey's post hoc analysis. ${ }^{*} P<0.05$, ${ }^{*} P<0.01,{ }^{* *} P<0.001$ between the indicated groups

dependent pathway via the activation of PI3K/Akt downstream and an insulin-independent pathway via AMPK $\alpha$ (Fig. 2). We found that 20E treatment can activate pAkt $\mathrm{Ser}^{473}$, which is the IRS substrate in the insulin signaling pathway. The activation of Akt resulted in the inhibition of FOXO1 translocation into the nucleus through pFOXO1 $\mathrm{Ser}^{256}$. FOXO1 is an important protein that activates PEPCK and G6Pase gene expression; thus, the inhibition of FOXO1 caused a reduction in hepatic gluconeogenesis. 20E also acted through insulinindependent pathways, such as through its action on AMPK $\alpha$. 20E activated AMPK $\alpha$ via pAMPK $\alpha \mathrm{Thr}^{172}$ (Fig. 2), which in turn suppressed CREB activity and helped stabilize the status and action of FOXO1 in the nucleus. Additionally, as the level of FGF21 expression was increased in the $20 \mathrm{E}$ treatment group (Fig. 2), FGF21 may contribute to the suppressive effect of $20 \mathrm{E}$ on hepatic glucose production. Even though the function of FGF21 has not been well established, a recent study suggested that FGF21 may regulate energy homeostasis through activation of the AMP-activated protein kinase (AMPK) signaling pathway [31]. These results further indicate that $20 \mathrm{E}$ can directly improve hepatic function by suppressing hepatic gluconeogenesis in metabolic disorder rats (Fig. 4). Another important insulin-sensitive organ that helps maintain glucose homeostasis is skeletal muscle. Under feeding conditions, skeletal muscle responds to insulin by increasing muscle glucose transport activity (Fig. 3). However, our study did not find an improvement in muscle glucose transport activity in 20E- treated groups. Arguably due to the action of $20 \mathrm{E}$ in skeletal muscle glucose transport, 20E possibly exerted anabolic activity [30] rather than metabolic activity in skeletal muscle.

The improvement of glucose tolerance and hyperinsulinemia observed in this study may lead to further prevention of CMS phenotypic characteristics [32]. Our study showed that 20E helped normalize blood pressure in the OHFFD rats (Table 3). This observation is consistent with the finding that $20 \mathrm{E}$ played a protective role against high blood pressure in spontaneously hypertensive rats [31]. Furthermore, we observed that 20E treatment reduced body weight in rats with CMS without affecting caloric intake. Consistent with previous studies $[16,19,21]$, the effect of $20 \mathrm{E}$ on body weight could be explained by a reduction in abdominal fat accumulation. It should be noted that a reduction in body weight of 20E-treated rats was not associated with any changes in lean body mass, represented by the plantaris weight (Table 1). While the effect of $20 \mathrm{E}$ on the serum lipid profile is still controversial [16, 20], our results showed that $20 \mathrm{E}$ effectively reduced serum LDL-cholesterol levels without affecting HDL-cholesterol or total cholesterol levels in the OHFFD groups (Table 2).

The molecular mechanisms involved in the 20Einduced effects are not well identified. To date, there are no confirmatory reports about the exact nuclear receptor in mammals that homologs to the 20E nuclear receptor found in insects. Despite other human steroid hormones, $20 \mathrm{E}$ did not appear to activate the nuclear androgen
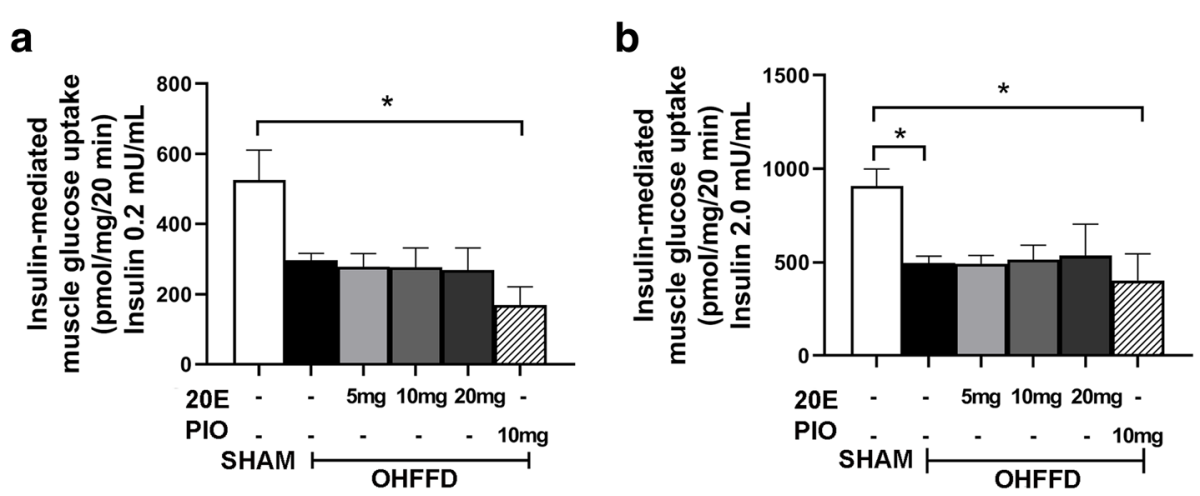

Fig. 3 Effects of 20-hydeoxyecdysone (20E; $5 \mathrm{mg} / \mathrm{kg}, 10 \mathrm{mg} / \mathrm{kg}, 20 \mathrm{mg} / \mathrm{kg}$ ) and pioglitazone (PIO) on insulin-mediated glucose transport under 2 insulin concentrations: (a) insulin-mediated muscle glucose uptake at $0.2 \mathrm{mU} / \mathrm{mL}$ insulin concentration, (b) insulin-mediated muscle glucose uptake at $2 \mathrm{mU} / \mathrm{mL}$ insulin. Values are means \pm SEM for 3-4 animals/group. Data comparisons between groups were performed using one-way ANOVA followed by Tukey's post hoc analysis. ${ }^{*} P<0.05$ between the indicated groups 


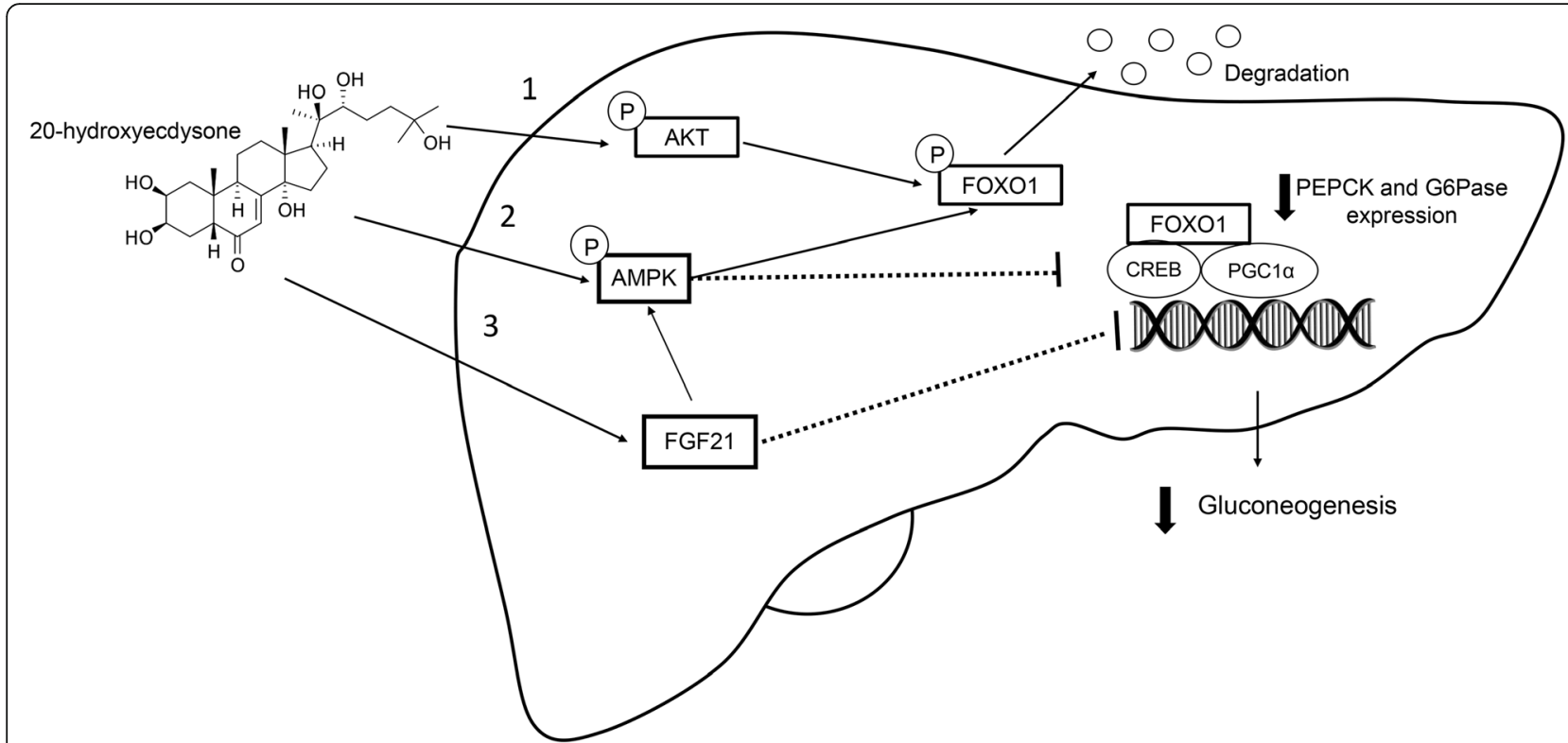

Fig. 4 Proposed mechanisms of 20-hydroxyecdysone action in the liver. 20E increases hepatic function by decreasing hepatic gluconeogenesis via both insulin-dependent downstream PI3K/Akt activation and insulin-independent pathways. 20E activates pAkt Ser ${ }^{473}$, which is the IRS substrate in the insulin signaling pathway. The activation of Akt caused the inhibition of FOXO1 to translocate into the nucleus through pFOXO1 $\mathrm{Ser}^{256}$. FOXO1 is an important protein that activates PEPCK and G6Pase gene expression. The inhibition of FOXO1 causes a reduction in hepatic gluconeogenesis. 20E also activates AMPKa via pAMPKa Thr ${ }^{172}$, which causes the suppression of CREB action that normally helps stabilize FOXO1 action inside the nucleus. 20E increases FGF21 expression, which then leads to the activation of pAMPKa Thr ${ }^{172}$

receptor (AR), even though the structure is similar to that of androgen steroids [11]. Alternatively, 20E might interact with receptors located on the cell membrane of mammalian cells. Antioxidative effects of $20 \mathrm{E}$ on cell membrane lipids have been described [33]. In addition, it has been shown that 20E stimulates the PI3K/AKT signal transduction pathway, which is also activated by insulin-like growth factor 1 (IGF-1). Although calciumdependent G-protein-coupled mechanism has also been suggested, the associated G-protein has not yet been characterized [34, 35]. The most recent finding proposed that 20E might have an anti-androgenic effect and that it acts through ER $\beta$ [36], which was shown in cell culture experiments and induced the hypertrophy of $\mathrm{C} 2 \mathrm{C} 12$ cells $[33,34]$. ER $\alpha$ is predominantly expressed in reproductive tissues such as the uterus and mammary glands, as well as in the kidney, liver, and heart. Contrary, ER $\beta$ is mostly expressed in vascular endothelial cells, the gastrointestinal tract, and the prostate [37]. Nevertheless, both receptors can be coexpressed in a number of tissues and cell types such as the thyroid, epididymis, bone, and regions of the brain. Expression of both ER $\alpha$ and ER $\beta$ are demonstrated in the skeletal muscle of mice $[38,39]$ and humans $[40,41]$. Consequently, selective activation of ER $\beta$ did not result in any classic estrogen action in this study. 20E did not result in common signs of classic estrogen activity, such as uterotrophy (Table 1). In addition, we found a diabetogenic effect of ER $\beta$ activation in skeletal muscle, as there was no improvement in skeletal muscle insulin-mediated glucose transport in 20E-treated rats. Our results support the theory that 20E may act through ER $\beta$ in mammals.

Broad-target pharmacological agents that modify insulin resistance are a good initial choice for CMS patients. PIO, a commonly prescribed drug for CMS patients, has been proven to be effective in alleviating metabolic dysfunction $[9,10]$. PIO is a synthetic ligand of peroxisome proliferator-activated receptor- $\gamma$ (PPAR $\gamma$ ), which is a member of the nuclear hormone receptor family and contributes to many biological processes, such as glucose regulation and lipid metabolism. We observed that PIO can alleviate metabolic dysfunction by improving whole body insulin sensitivity via the reduction of hepatic glucose production but independent of the action of skeletal muscle glucose transport. The atypical effect of PIO on skeletal muscle glucose transport in this study may be due to the expression of PPARy being lower in skeletal muscle than in liver and adipose tissue [10]. In this study, PIO prevented the development of other CMS phenotypes, such as abdominal fat accumulation, elevated LDL-cholesterol and triglyceride levels, and high blood pressure. In comparison to 20E, PIO had better efficacy in regulating the whole body lipid profile, especially triglycerides, while $20 \mathrm{E}$ had a better ability to activate FGF21 expression in the liver presumably causing suppression of hepatic glucose production in 
OHFFD rats. Therefore, the extent of insulin resistance reduction and prevention of CMS characteristics in PIOtreated OHFFD rats is somewhat similar to that associated with $20 \mathrm{E}$ treatment.

In summary, our findings emphasize the favorable effects of 20E treatment against CMS. 20E mediates improvements in whole-body insulin sensitivity by controlling whole-body glucose homeostasis by modulating hepatic expression, contributing to the proper maintenance of glucose homeostasis, lipid profile, and arterial blood pressure. Therefore, the present study indicates that 20E may be considered an alternative choice for CMS prevention or alleviation of metabolic risk factors beyond lifestyle changes, and further information should be acquired.

\section{Conclusion}

Our findings indicate the beneficial effects of $20 \mathrm{E}$ treatment against CMS. 20E treatment proved to be effective in improving whole-body insulin sensitivity by controlling whole-body glucose homeostasis by activating protein expression in the liver (pAkt $\mathrm{Ser}^{473}$, pFOXO1 $\mathrm{Ser}^{256}$, pAMPK $\alpha \mathrm{Thr}^{172}$, and FGF21). In addition, $20 \mathrm{E}$ helps control serum LDL cholesterol levels and maintain arterial blood pressure. Therefore, this study demonstrated that 20E may be an alternative for CMS prevention or treatment. It should be noted that an absence of beneficial effects of $20 \mathrm{E}$ on insulin-stimulated glucose transport reported in the present study was performed on the soleus muscle (mainly slow twitch fibers). Accordingly, future investigation is required to verify whether the same responses will be observed in other insulin-sensitive tissues, such as adipocytes or muscles with different fiber type composition.

\section{Abbreviations}

20E: 20-hydroxyecdysone; CMS: Cardiometabolic syndrome;

OHFFD: Ovariectomized rats fed a high-fat-high-fructose diet;

PIO: Pioglitazone; LDL: low-density lipoprotein-cholesterol; HDL: High-density lipoprotein-cholesterol; TC: Total cholesterol; SBP: Systolic blood pressure; DBP: Diastolic blood pressure; MAP: Mean arterial blood pressure; 2-DG: 2deoxy- $\left[^{3} \mathrm{H}\right]$-glucose; KHB: Krebs-Henseleit buffer; BSA: Bovine serum albumin; TBS: Tris-buffered saline; HOMA-IR: Homeostatic Model Assessment of Insulin Resistance; G-I index: Glucose-insulin index; AUC: Area under the curve; OGTT: Oral glucose tolerance test

\section{Acknowledgments}

The authors thank Prof. Apichart Suksamrarn, Department of Chemistry, Faculty of Science, Ramkhamheang University, Bangkok, Thailand that kindly provided 20E for this research.

\section{Authors' contributions}

$J B, J W, T B$, and VS designed the experiment. JB treated the animals. JB, NC, YR, JS and VS collected animal blood and tissues and performed laboratory measurements. JB and VS analyzed and interpreted the data. JB and VS were major contributors to the writing of the manuscript. All authors drafted, improved, and revised the manuscript. All authors read and approved the final manuscript.

\section{Funding}

This work was supported by grants from Mahidol University and Thailand Research Fund through the Royal Golden Jubilee Ph.D. program (PHD/4.PE. $\mathrm{MU} / 56 / \mathrm{C} .1$ ) to JB and VS. The funders did not have any role in study design, data collection, management, analysis, data interpretation, manuscript writing, and the decision to submit the manuscript for publication.

\section{Availability of data and materials}

The datasets used and/or analyzed during the current study are available from the corresponding author on reasonable request.

\section{Ethics approval and consent to participate}

The animal protocol (No. MUSC58-007-322) was approved by the Institutional Animal Care and Use Committee of the Faculty of Science, Mahidol University in accordance with the International Guiding Principles for Biomedical Research Involving Animals of the Council for International Organizations of Medical Sciences.

\section{Consent for publication}

Not applicable.

\section{Competing interests}

The authors declare no competing interests.

\section{Author details}

${ }^{1}$ Department of Physiology, Faculty of Science, Mahidol University, Bangkok 10400, Thailand. 'Faculty of Physical Therapy, Huachiew Chalermprakiet University, Samut Prakan 10540, Thailand. 'Division of Physical Therapy, Faculty of Physical Therapy, Srinakharinwirot University, Nakhon Nayok 26120, Thailand. ${ }^{4}$ Faculty of Physical Therapy, Mahidol University, Nakhonpathom 73170, Thailand.

Received: 29 January 2020 Accepted: 27 April 2020

Published online: 06 May 2020

\section{References}

1. Grassi G, Seravalle G, Quarti-Trevano F, Dell'Oro R, Bombelli M, Mancia G. Metabolic syndrome and cardiometabolic risk: an update. Blood Press. 2009; 18(1-2):7-16.

2. Grundy SM. Metabolic syndrome update. Trends Cardiovasc Med. 2016; 26(4):364-73.

3. Murase T, Mizuno T, Omachi T, Onizawa K, Komine Y, Kondo H, et al. Dietary diacylglycerol suppresses high fat and high sucrose diet-induced body fat accumulation in C57BL/6J mice. J Lipid Res. 2001;42(3):372-8.

4. Panchal SK, Brown L. Rodent models for metabolic syndrome research. J Biomed Biotechnol. 2011;2011:351982.

5. Panchal SK, Poudyal H, lyer A, Nazer R, Alam MA, Diwan V, et al. Highcarbohydrate, high-fat diet-induced metabolic syndrome and cardiovascular remodeling in rats. J Cardiovasc Pharmacol. 2011;57(5):611-24.

6. Parekh PI, Petro AE, Tiller JM, Feinglos MN, Surwit RS. Reversal of dietinduced obesity and diabetes in C57BL/6J mice. Metabolism. 1998;47(9): 1089-96.

7. Carr MC. The emergence of the metabolic syndrome with menopause. J Clin Endocrinol Metab. 2003:88(6):2404-11.

8. Farahmand M, Ramezani Tehrani F, Bahri Khomami M, Noroozzadeh M, Azizi F. Surgical menopause versus natural menopause and cardio-metabolic disturbances: a 12-year population-based cohort study. J Endocrinol Investig. 2015;38(7):761-7.

9. Gupta P, Bala M, Gupta S, Dua A, Dabur R, Injeti E, et al. Efficacy and risk profile of anti-diabetic therapies: conventional vs traditional drugs-a mechanistic revisit to understand their mode of action. Pharmacol Res. 2016;113(Pt A):636-74.

10. Krentz AJ, Bailey CJ. Oral antidiabetic agents: current role in type 2 diabetes mellitus. Drugs. 2005;65(3):385-411.

11. Bathori $M$, Pongracz Z. Phytoecdysteroids--from isolation to their effects on humans. Curr Med Chem. 2005;12(2):153-72.

12. Dinan L. Phytoecdysteroids: biological aspects. Phytochemistry. 2001;57(3): 325-39.

13. Dinan L. The Karlson lecture. Phytoecdysteroids: what use are they? Arch Insect Biochem Physiol. 2009;72(3):126-41. 
14. Lafont R, Dinan L. Practical uses for ecdysteroids in mammals including humans: an update. J Insect Sci. 2003;3:7.

15. Dinan $L$, Lafont R. Effects and applications of arthropod steroid hormones (ecdysteroids) in mammals. J Endocrinol. 2006;191(1):1-8.

16. Toth N, Szabo A, Kacsala P, Heger J, Zador E. 20-Hydroxyecdysone increases fiber size in a muscle-specific fashion in rat. Phytomedicine. 2008;15(9):691-8.

17. Chermnykh NS, Shimanovskii NL, Shutko GV, Syrov VN. The action of methandrostenolone and ecdysterone on the physical endurance of animals and on protein metabolism in the skeletal muscles. Farmakol Toksikol. 1988;51(6):57-60.

18. Seidlova-Wuttke D, Ehrhardt C, Wuttke W. Metabolic effects of 20-OH-ecdysone in ovariectomized rats. J Steroid Biochem Mol Biol. 2010;119(3-5):121-6.

19. Chen $Q, X i a$ Y, Qiu Z. Effect of ecdysterone on glucose metabolism in vitro. Life Sci. 2006;78(10):1108-13.

20. Yoshida T, Otaka T, Uchiyama M, Ogawa S. Effect of ecdysterone on hyperglycemia in experimental animals. Biochem Pharmacol. 1971;20(12): 3263-8.

21. Foucault AS, Mathe V, Lafont R, Even P, Dioh W, Veillet S, et al. Quinoa extract enriched in 20-hydroxyecdysone protects mice from diet-induced obesity and modulates adipokines expression. Obesity (Silver Spring). 2012 20(2):270-7.

22. Naresh Kumar R, Sundaram R, Shanthi P, Sachdanandam P. Protective role of 20-OH ecdysone on lipid profile and tissue fatty acid changes in streptozotocin induced diabetic rats. Eur J Pharmacol. 2013;698(1-3):489-98,

23. Kizelsztein P, Govorko D, Komarnytsky S, Evans A, Wang Z, Cefalu WT, et al. 20-Hydroxyecdysone decreases weight and hyperglycemia in a dietinduced obesity mice model. Am J Physiol Endocrinol Metab. 2009;296(3): E433-9.

24. Dushkin M, Khrapova M, Kovshik G, Chasovskikh M, Menshchikova E, Trufakin $V$, et al. Effects of rhaponticum carthamoides versus glycyrrhiza glabra and punica granatum extracts on metabolic syndrome signs in rats. BMC Complement Altern Med. 2014;14:33.

25. Buniam J, Chukijrungroat N, Khamphaya T, Weerachayaphorn J, Saengsirisuwan V. Estrogen and voluntary exercise attenuate cardiometabolic syndrome and hepatic steatosis in ovariectomized rats fed a high-fat high-fructose diet. Am J Physiol Endocrinol Metab. 2019;316(5): E908-21.

26. Prasannarong M, Saengsirisuwan V, Piyachaturawat P, Suksamrarn A. Improvements of insulin resistance in ovariectomized rats by a novel phytoestrogen from Curcuma comosa Roxb. BMC Complement Altern Med. 2012;12:28.

27. Rattanavichit Y, Chukijrungroat N, Saengsirisuwan V. Sex differences in the metabolic dysfunction and insulin resistance of skeletal muscle glucose transport following high fructose ingestion. Am J Physiol Regul Integr Comp Physiol. 2016;311(6):R1200-12.

28. Henriksen EJ, Halseth AE. Early alterations in soleus GLUT-4, glucose transport, and glycogen in voluntary running rats. J Appl Physiol (1985). 1994;76(5):1862-7.

29. Cooke PS, Spencer TE, Bartol FF, Hayashi K. Uterine glands: development, function and experimental model systems. Mol Hum Reprod. 2013;19(9): 547-58.

30. Sundaram R, Naresh R, Shanthi P, Sachdanandam P. Efficacy of 20-OHecdysone on hepatic key enzymes of carbohydrate metabolism in streptozotocin induced diabetic rats. Phytomedicine. 2012;19(8-9):725-9.

31. Chau MD, Gao J, Yang Q, Wu Z, Gromada J. Fibroblast growth factor 21 regulates energy metabolism by activating the AMPK-SIRT1-PGC-1alpha pathway. Proc Natl Acad Sci U S A. 2010;107(28):12553-8.

32. Tran LT, Yuen VG, McNeill JH. The fructose-fed rat: a review on the mechanisms of fructose-induced insulin resistance and hypertension. Mol Cell Biochem. 2009:332(1-2):145-59.

33. Parr MK, Zhao P, Haupt O, Ngueu ST, Hengevoss J, Fritzemeier KH, et al. Estrogen receptor beta is involved in skeletal muscle hypertrophy induced by the phytoecdysteroid ecdysterone. Mol Nutr Food Res. 2014;58(9):1861-72.

34. Gorelick-Feldman J, Cohick W, Raskin I. Ecdysteroids elicit a rapid Ca2+ flux leading to Akt activation and increased protein synthesis in skeletal muscle cells. Steroids. 2010;75(10):632-7.

35. Gorelick-Feldman J, Maclean D, llic N, Poulev A, Lila MA, Cheng D, et al. Phytoecdysteroids increase protein synthesis in skeletal muscle cells. J Agric Food Chem. 2008:56(10):3532-7.
36. Phungphong S, Kijtawornrat A, Chaiduang S, Saengsirisuwan V, Bupha-Intr T. 20-Hydroxyecdysone attenuates cardiac remodeling in spontaneously hypertensive rats. Steroids. 2017;126:79-84.

37. Matthews J, Gustafsson JA. Estrogen receptor and aryl hydrocarbon receptor signaling pathways. Nucl Recept Signal. 2006;4:e016.

38. Milanesi L, de Boland AR, Boland R. Expression and localization of estrogen receptor alpha in the C2C12 murine skeletal muscle cell line. J Cell Biochem. 2008;104(4):1254-73.

39. Milanesi L, Vasconsuelo A, de Boland AR, Boland R. Expression and subcellular distribution of native estrogen receptor beta in murine $\mathrm{C} 2 \mathrm{C} 12$ cells and skeletal muscle tissue. Steroids. 2009;74(6):489-97.

40. Wiik A, Ekman M, Johansson O, Jansson E, Esbjornsson M. Expression of both oestrogen receptor alpha and beta in human skeletal muscle tissue. Histochem Cell Biol. 2009;131(2):181-9.

41. Wiik A, Glenmark B, Ekman M, Esbjornsson-Liljedahl M, Johansson O, Bodin $\mathrm{K}$, et al. Oestrogen receptor beta is expressed in adult human skeletal muscle both at the mRNA and protein level. Acta Physiol Scand. 2003; 179(4):381-7.

\section{Publisher's Note}

Springer Nature remains neutral with regard to jurisdictional claims in published maps and institutional affiliations.
Ready to submit your research? Choose BMC and benefit from:

- fast, convenient online submission

- thorough peer review by experienced researchers in your field

- rapid publication on acceptance

- support for research data, including large and complex data types

- gold Open Access which fosters wider collaboration and increased citations

- maximum visibility for your research: over $100 \mathrm{M}$ website views per year

At BMC, research is always in progress.

Learn more biomedcentral.com/submissions 